\title{
Characterizing an engineered carotenoid-producing yeast as an anti-stress chassis for building cell factories
}

\author{
Hsien-Lin Liu'1,34+, Jui-Jen Chang ${ }^{2,3 \dagger}$, Caroline Thia ${ }^{4}$, Yu-Ju Lin ${ }^{3}$, Shou-Chen Lo ${ }^{4}$, Chieh-Chen Huang ${ }^{1,4,5^{*}}$ \\ and Wen-Hsiung $\mathrm{Li}^{1,3,6^{*}}$
}

\begin{abstract}
Background: A microorganism engineered for non-native tasks may suffer stresses it never met before. Therefore, we examined whether a Kluyveromyces marxianus strain engineered with a carotenoid biosynthesis pathway can serve as an anti-stress chassis for building cell factories.

Results: Carotenoids, a family of antioxidants, are valuable natural products with high commercial potential. We showed that the free radical removal ability of carotenoids can confer the engineered host with a higher tolerance to ethanol, so that it can produce more bio-ethanol than the wild type. Moreover, we found that this engineered strain has improved tolerance to other toxic effects including furfurals, heavy metals such as arsenate (biomass contaminant) and isobutanol (end product). Furthermore, the enhanced ethanol tolerance of the host can be applied to bioconversion of a natural medicine that needs to use ethanol as the delivery solvent of hydrophobic precursors. The result suggested that the engineered yeast showed enhanced tolerance to ethanol-dissolved hydrophobic 10-deacetylbaccatin III, which is considered a sustainable precursor for paclitaxel (taxol) bioconversion.
\end{abstract}

Conclusions: The stress tolerances of the engineered yeast strain showed tolerance to several toxins, so it may serve as a chassis for cell factories to produce target products, and the co-production of carotenoids may make the biorefinary more cost-effective.

Keywords: Carotenoids, Toxins, Bio-ethanol, Anti-stress, 10-deacetylbaccatin III, Anti-stress, Biorefinery

\section{Background}

Due to the increasing demand of alternative fuel and biopharmaceuticals, people are looking for reliable and sustainable ways to produce various bio-products. Synthetic biology, which can be used to design and integrate new biological functions into a cell, provides a powerful way to engineer a microbe for a sustainable bio-industry. Its broad applications can incorporate desirable bio-processes into a designer host to convert

*Correspondence: cchuang@dragon.nchu.edu.tw; whli@uchicago.edu ${ }^{\dagger}$ Hsien-Lin Liu and Jui-Jen Chang contributed equally to this work

${ }^{1}$ Ph.D. Program in Microbial Genomics, National Chung Hsing University and Academia Sinica, Taipei, Taiwan

${ }^{6}$ Department of Ecology and Evolution, University of Chicago, Chicago, IL 60637, USA

Full list of author information is available at the end of the article biomass to valuable bio-products such as biofuels [1]. Moreover, producing multiple bio-products simultaneously in a single microbe, such as co-production of biofuel and valuable natural products, can achieve competitive advantages for a multipurpose biorefinery $[2,3]$. However, the toxic effects from precursors, intermediate products or end products can cause a physiological imbalance to the host. Exposure to xenobiotics may lead to an increase of reactive oxygen species (ROS) and free radicals intracellularly, which can cause cellular damages $[4,5]$. The cellular membrane is an important boundary to protect cells from external stresses, but excessive free radicals can attack the membrane by lipid peroxidation. Previously, metallothioneins, which are the membrane-targeted antioxidative proteins, were demonstrated to improve the cell 
tolerance against $n$-butanol by scavenging intracellular or extracellular ROS [6, 7]. Furthermore, n-butanol production was increased via co-expression of metallothioneins [8]. The results implied that the ROS scavenging capacity of the host cell is important for cell factory development.

In this study, the yeast Kluyveromyces marxianus was used as the host because it has several desirable characteristics for industrial applications. First, $K$. marxianus is a Crabtree-negative yeast that exhibits enhanced biomass production when supplementing with excessive glucose. Second, K. marxianus is capable of fermentation at a broad range of temperatures ( 25 to $\sim 45^{\circ} \mathrm{C}$ ). Third, $K$. marxianus can utilize various carbon sources, including lactose, xylose, arabinose, cellobiose, and inulin. Finally, K. marxianus is GRAS (generally regarded as safe) and QPS (qualified presumption of safe) and has been widely employed in various biotechnological applications and food industry [9].

In our previous study, a carotenoid biosynthesis pathway was integrated into K. marxianus [10]. Carotenoids are superior antioxidants, which have an ability to neutralize singlet oxygen and to protect cellular membranes from ultraviolet (UV) light and toxic oxidative stresses [11]. Moreover, carotenoids have been used in the prevention of various human diseases [12, $13]$ and may act as value-added products.

In this study, we tested if our carotenoid-producing $K$. marxianu strains can tolerate toxic compounds that may appear in biofuel production. As consolidated bioprocessing (CBP) provides a simple way to integrate biomass hydrolysis and fermentation in one process, furfurals are often generated from lignocellulosic biomass hydrolysis and inhibit yeast growth. In addition, heavy metals may be present when utilizing biomass harvested from polluted environment. Increasing the tolerance of the host to heavy metals may enhance its ability to utilize biomass from phytoremediation. Phytoremediation may provide a cost-effective way to cleanup heavy metals in contaminated soil and water by plants, and the biomass can then be a source for renewable energy and bio-products [14]. Therefore, tolerance assays were conducted to evaluate the potential of carotenoids producing strain for biofuel production that may face ethanol, furfurals and heavy metals. In addition, we also tested the tolerance of the carotenoid-producing strain to ethanol dissolved with 10-deacetylbaccatin III, which is a precursor to paclitaxel, an anti-cancer medicine. Thus, this study explored the potential of using a carotenoid-producing strain as a chassis for constructing multipurpose cell factories.

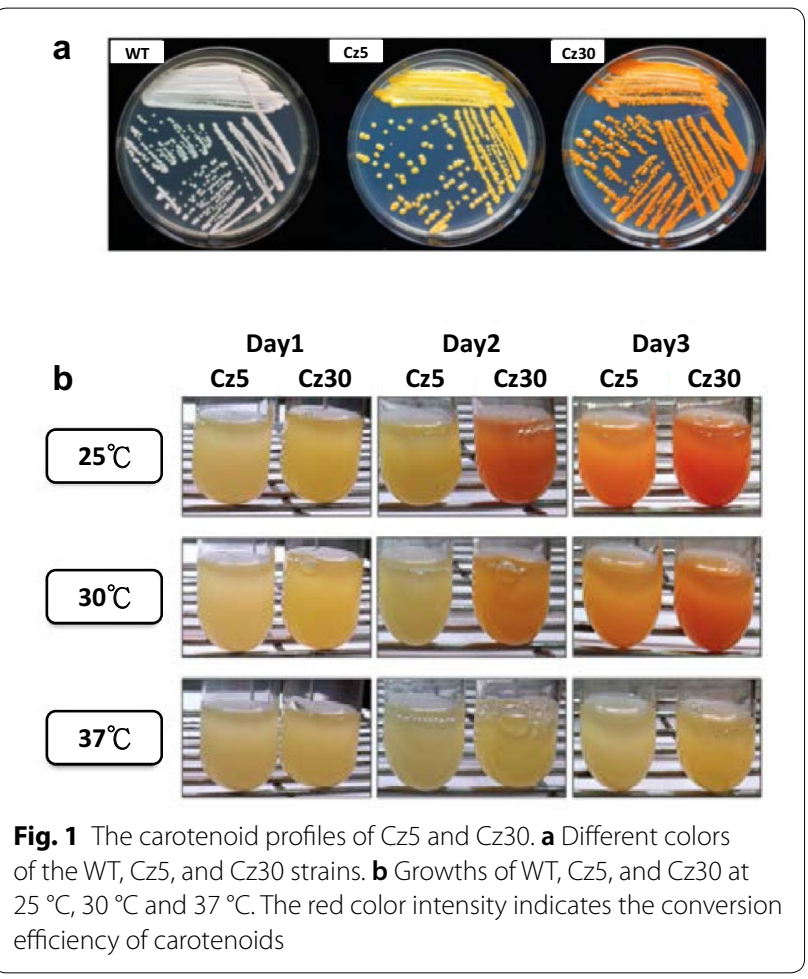

\section{Results}

\section{Characterization of two engineered strains}

Our carotenoid-biosynthesis pathway includes the truncated 3-hydroxy-3-methylglutaryl -coenzyme A reductase (tHMG1 gene) from Kluyveromyces marxianus, the geranylgeranyl pyrophosphate synthase ( $c r t E$ gene) from Xanthophyllomyces dendrorhous, the $\operatorname{cr} t Y B$ gene (phytoene synthase/lycopene cyclase) of $X$. dendrorhous, the crtI gene (phytoene desaturase) of $X$. dendrorhous, the $\beta$-carotene ketolase (bkt gene) from Chlamydomonas reinhardtii, and the $\beta$-carotene hydroxylase (chyb gene) from Chlorella zofingiensis. In a previous study [10], the synthetic biology tool PGASO (Promoter-based Gene Assembly and Simultaneous Overexpression) [15] was employed to integrated the pathway into the host genome, and the seven gene cassettes (promotergene-terminator) including KIPLac4-crtI-KITTLac4, ScPGapDH-crtE-ScTTGap, ScPGK-chyb-ScTTPGK, KlPGapDH-kanMX-ScTTGap, KIPGK-bkt-ScTTPGK, KIPADHI-crtYB-ScTTGap, and ScPADHI- $t H M G-$ ScTTADHI, were co-transformed into the yeast host, $K$. marxianus. As the colony color was correlated with the carotenoids amount produced, one of the light red colonies was selected and denoted as Cz5 strain and the reddest colony was selected and denoted as the Cz30 strain.

In this study, we characterized the two engineered strains by color observation, transcription measurement, and metabolite analysis. Compared to the wild type 
(WT) strain, the Cz30 strain exhibited stronger red color than Cz5, while WT did not show red color (Fig. 1a). The yellow (Cz5) and red orange color (Cz30) implies the existence of carotenoids. The gene expression profiles of $\mathrm{Cz} 5$ and $\mathrm{Cz} 30$ at different growth temperatures $\left(25^{\circ} \mathrm{C}, 30^{\circ} \mathrm{C}\right.$, and $\left.37^{\circ} \mathrm{C}\right)$ were examined by quantitative reverse transcription PCR (RT-qPCR). Since phytoene desaturase (encoded by $c r t I$ ) and $\beta$-carotene ketolase (encoded by $B K T$ ) are the crucial enzymes in the production of $3 \mathrm{~S}, 3^{\prime} \mathrm{S}$-astaxanthin, two stronger promoters, pLac4 and pKIPGK, were used to drive these two genes. Accordingly, the RT-qPCR data indicated that the expression levels of the CrtI and CrBKT genes were higher than those of the other genes (Fig. 1b). Furthermore, all carotenoid-biosynthesis pathway genes of $\mathrm{Cz} 30$ showed higher expression levels than those of $\mathrm{Cz} 5$. Among the three growth temperatures $\left(25^{\circ} \mathrm{C}, 30^{\circ} \mathrm{C}\right.$ and $\left.37^{\circ} \mathrm{C}\right)$, the strongest color was found at $25^{\circ} \mathrm{C}$ (Additional file 1: Fig. S1). The carotenoids profiles of $\mathrm{Cz} 5$ and $\mathrm{Cz} 30$ were further verified by HPLC spectrometry under UV460 nm. Carotenoids were detected in both $\mathrm{Cz} 5$ and $\mathrm{Cz} 30$, but not in WT (Additional file 1: Fig. S2). The total amount of carotenoids of Cz5 was $\sim 137.2 \mu \mathrm{g} / \mathrm{g}$ [10], while that of $\mathrm{Cz} 30$ was $\sim 250.5 \mu \mathrm{g} / \mathrm{g}$. The data indicated that the Cz30 strain produced higher amounts of carotenoids than $\mathrm{Cz} 5$, and was chosen for anti-stress assays.

\section{Enhancement of free-radical scavenging capacity and cell protection ability}

To determine the free radical scavenging capacity, the crude extract of $\mathrm{Cz} 30$ was used to evaluate the antioxidant ability using 2,2'-azino-bis (3-ethylbenzothiazoline6-sulphonic acid) (ABTS) reaction and Trolox Equivalent Antioxidant Capacity (TEAC) assay. The extract of Cz30 showed a higher free radical scavenging capacity (72.1\%) than that of WT $(52.3 \%)$ by ABTS per $2 \mathrm{mg}$ dry cell weight (Fig. 2a). For the TEAC assay, the antioxidant capacity of the Cz30 extract per gram dry cell weight was $1.95 \mathrm{mg}$ of Trolox, while that of the WT extract was only $1.41 \mathrm{mg}$ of Trolox (Fig. 2b). Thus, the carotenoids of the Cz30 strain might can increase $\sim 38 \%$ free radicals scavenging capacity in yeast host.

To test the ability of carotenoids to prevent the cellular damage from UV light, the engineered strains were exposed to UV radiation for 5, 10, or $20 \mathrm{~min}$. The survival rate was measured by the colony number counting. Cz30 showed a better survival rate than WT (Fig. 2c). Apparently, the antioxidant activity of carotenoids reduced the mortality of Cz30 from UV damage.

\section{Improving alcohol production}

In the survival assay, the serial dilution testing showed that Cz30 has a better survival rate than WT in



different concentrations of ethanol stress (Fig. 3a). Figure $3 \mathrm{~b}$ shows that at 2,4 , or $6 \%$ ethanol, the cell growth of WT was significantly more strongly repressed by ethanol than that of $\mathrm{Cz} 30$. Moreover, $\mathrm{Cz} 30$ produced more ethanol (3.5\%) compared to WT (2.5\%) after $72 \mathrm{~h}$ (Fig. 3c). Figure 3d-f showed the growth curves of different strains. These data indicated that carotenoids 


\section{a}

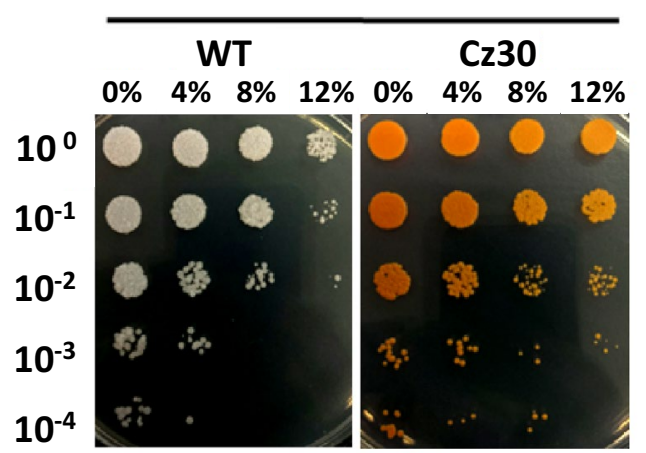

C

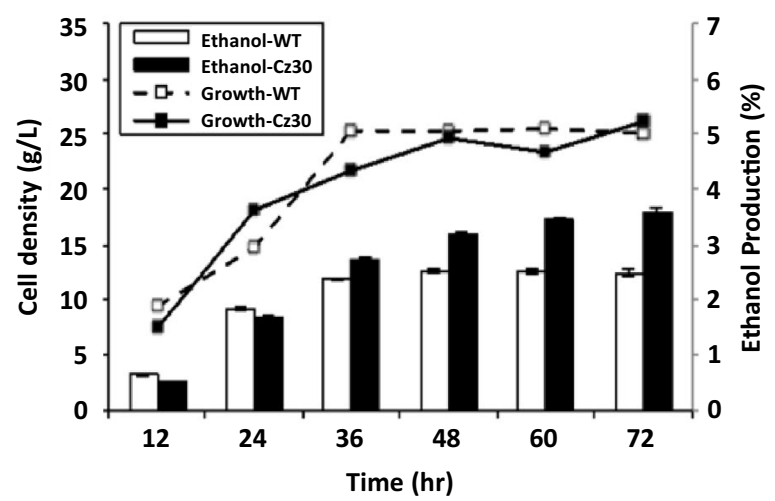

e

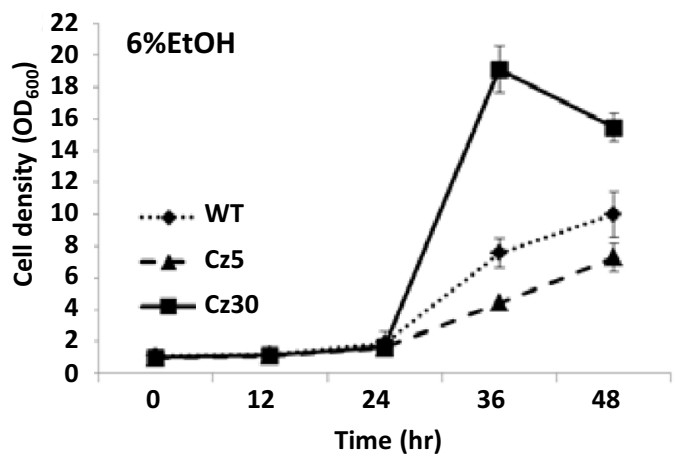

b

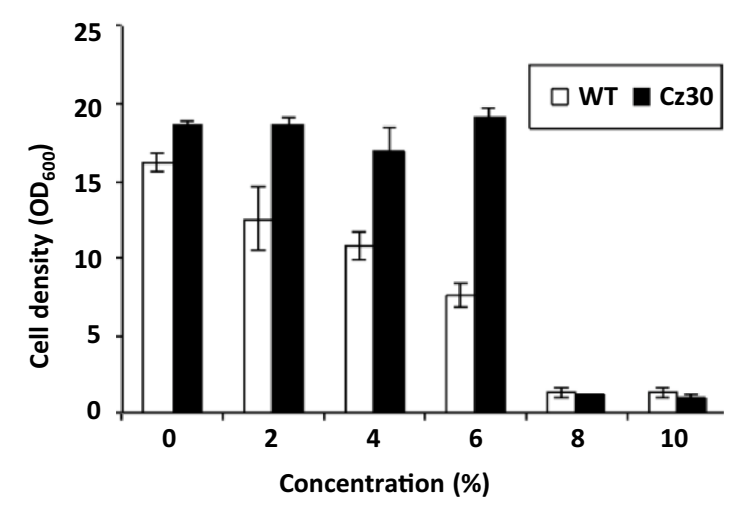

d

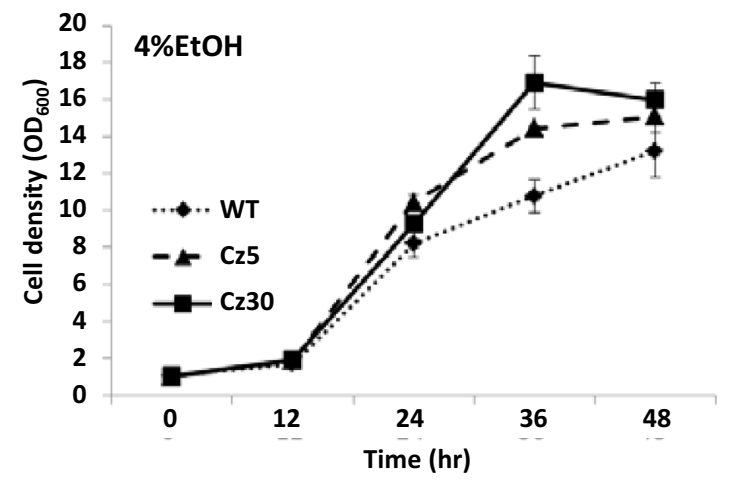

f

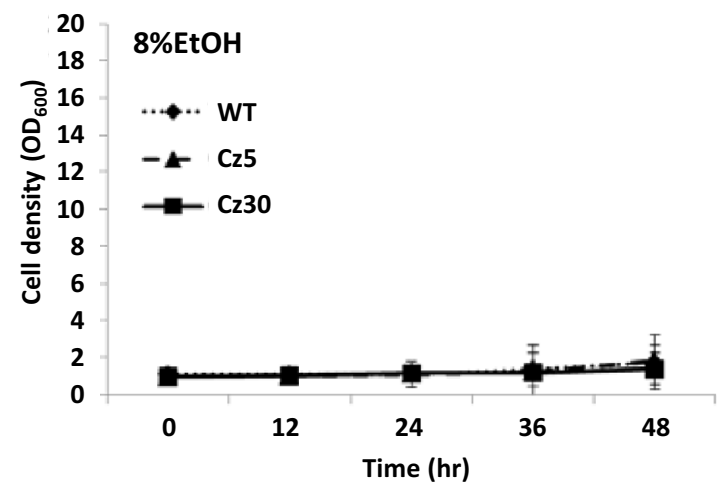

Fig. 3 Alcohol tolerance and production of WT, Cz30 and Cz5. a The survival assay. The colonies of WT and Cz30 were exposed to 0\%, 4\%, 8\% and $12 \%$ ethanol concentrations. b The growth assay. The cell densities of WT and Cz30 strains treated with different ethanol concentrations were measured at 36 h. Cz30 showed better growth than WT at 2\%, 4\% and 6\% ethanol concentrations. c Cell growth and ethanol production of WT and $\mathrm{Cz} 30$ strains. The left y axis represents the cells density, the right $y$ axis represents the ethanol production, and the $\mathrm{x}$ axis represents the time. Cz30 showed a higher ethanol production than WT. d-f WT, Cz5, and Cz30 were exposed to 4\%, 6\%, and 8\% ethanol for 48 h in growth assay. Cz30 exhibited higher tolerance than Cz5 and WT at 4\% and 6\% ethanol. Cz5 exhibited better tolerance than WT at 4\%, but not at 6\%. WT, Cz5, and Cz30 did not grow at $8 \%$ ethanol at the initial cell density of $1.0 \mathrm{OD}_{600 \mathrm{~nm}}$. The data represent the mean $\pm \mathrm{SD}(\mathrm{n}=3)$

might protect the host from the damage by ethanol during the fermentation process and improved the ethanol production. It also showed that $\mathrm{Cz} 30$ can be engineered to convert biomass to valuable carotenoids and ethanol simultaneously. 


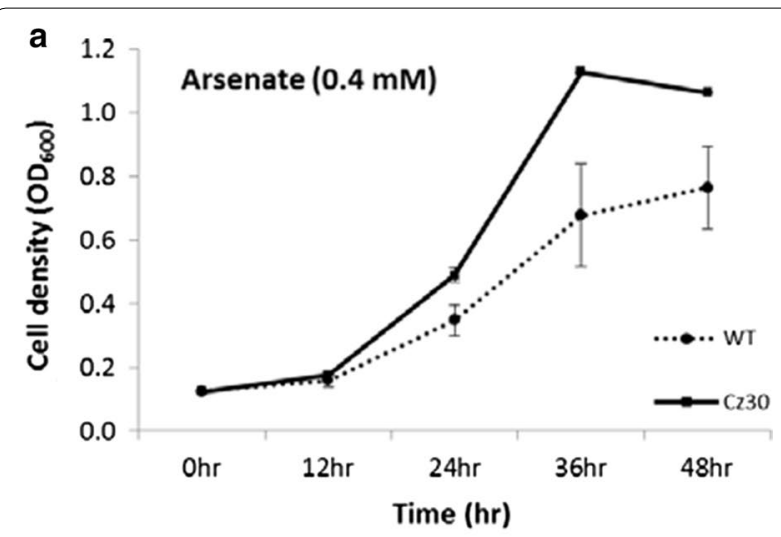

b

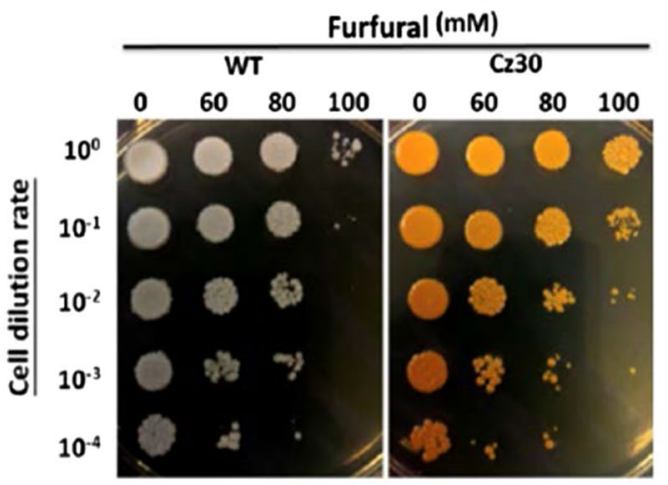

C

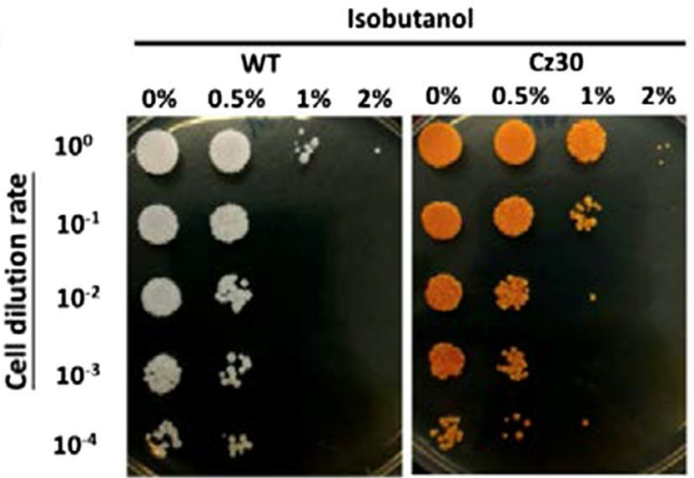

Fig. 4 Growth assays under assenate, furfural and isobutanol. Cz30 grew better than WT under $0.4 \mathrm{mM}$ arsenate (a). The serial dilutions after various titer exposures to furfural (b) and isobutanol (c) for $24 \mathrm{~h}$ showed a better survival rate for $\mathrm{Cz} 30$ than WT. The data represent the mean $\pm S D(n=3)$

\section{Improvement of stress tolerance}

Furfural and heavy metal arsenate $\left(\mathrm{As}[\mathrm{V}], \mathrm{AsO}_{4}{ }^{3-}\right)$ can cause an oxidative stress in yeast $[16,17]$. Furthermore, biofuels, such as isobutanol, can also cause stresses to yeast cells. The engineered strains were therefore tested for its ability to tolerate arsenate, furfural, and isobutanol. Compared to WT, Cz30 showed better tolerances to arsenate, furfural, and isubutanol (Fig. $4 \mathrm{a}-\mathrm{c}$ ). Hence, the production of carotenoids might protect $\mathrm{Cz} 30$ from various toxins.

\section{Improved tolerance of Cz30 to 10-deacetyl baccatin III}

The metabolite baccatin III is a crucial precursor for semi-synthesis of paclitaxel and its derivatives. The metabolite10-deacetyl baccatin III (10-DB III) is the natural precursor of baccatin III, and it has a high concentration $(0.1 \%)$ in needle extracts of the common ornamental yew (Taxus baccata) [18], and therefore has been considered a cost-efficient and eco-friendly source. However, ethanol is an important solvent for dissolving this hydrophobic precursor. Due to the saturation solubility of 10-DB III in ethanol $(20 \mathrm{mM})$, the ethanol tolerance became a limitation of substrate supplementation, and the improvement of ethanol tolerance could be helpful to overcome this obstacle. Hence, the tolerance of Cz30 was analyzed by treatment of ethanol-dissolved 10-deacetyl baccatin III. In survival assays, WT and Cz30 were exposed to $0,0.8,1.6$ or $3.2 \mathrm{mM}$ of 10 -deacetyl baccatin III that were dissolved in $0,4,8$ or $12 \%$ ethanol for $24 \mathrm{~h}$, and subsequently inoculated into the YPG plate with a series dilution. The result showed that Cz30 had a better survival rate than WT (Fig. 5a). It was also subjected to the growth assay in YPG medium with different initial concentrations of ethanol supplemented with/without 10-DB III. The Cz30 showed better growth in ethanol supplemented with or without 10-DB III than WT (Fig. 5b). These results were supported by the growth curve assay under $0.8 \mathrm{mM}$ of 10 -deacetylbaccatin III with $4 \%$ ethanol and $1.2 \mathrm{mM}$ of 10 -deacetylbaccatin III with $6 \%$ ethanol (Fig. $5 \mathrm{c}$, d). These data suggested that the carotenoids can protect the host cell from the damage by 10 -deacetylbaccatin III. Thus, carotenoid-producing yeast strains can help the conversion of 10-deacetylbaccatin III to paclitaxel or its derivatives.

\section{Discussion}

Simultaneously production of multiple bio-products with a good biorefinery approach may reduce cost. However, the host may confront more stresses. The tolerance to multiple stresses and/or toxins is a key challenge to improve the performance of bioconversion. Carotenoids are natural antioxidants that can help tolerate stresses and provide benefits to human health. This concept has here been shown by constructing a carotenoid-production yeast. By using the PGASO method, the target genes were integrated into the genome by homologous recombination that was targeted to the region of Lac4 promoter. The homologous sequence was modified from the Lac4 promoter, so that the integration did not disrupt the landing site but could create one more copy of the Lac4 promoter for further integration. This characteristic 


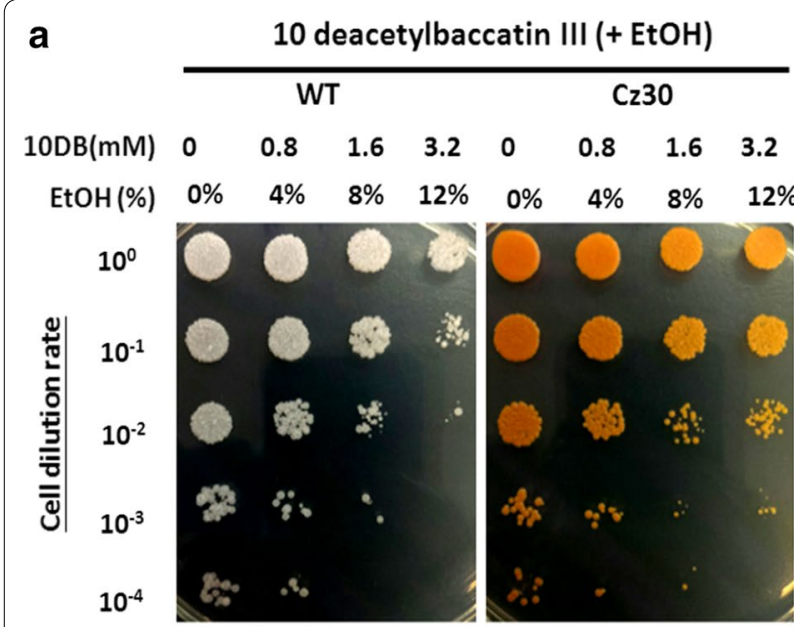

\section{b}

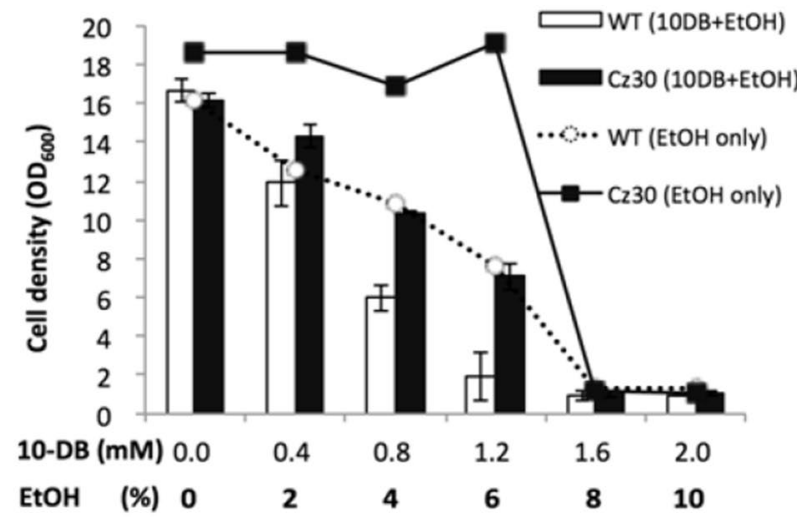

C


Fig. 5 Survival assays under 10-deacetylbaccatin III and ethanol. a The survival of the colonies of WT and Cz30 strains under 10 deacetylbaccatin III (10-DB III) and ethanol. b The cell densities of WT and Cz30 strains after growth for $36 \mathrm{~h}$ in various concentrations of ethanol mixed with/without 10-DB III. c, $\mathbf{d}$ The cell growth assays under $0.8 \mathrm{mM} 10-\mathrm{DB}$ III (+4\% EtOH) and $1.2 \mathrm{mM} \mathrm{10-DB} \mathrm{III} \mathrm{(+6 \%} \mathrm{EtOH),} \mathrm{respectively.} \mathrm{The} \mathrm{data} \mathrm{represent} \mathrm{the}$ mean $\pm S D(n=3)$

provided an opportunity for simultaneous multiple-integrations. Thus, different transgenic lines may carry different copy numbers of the transgenes. Based on the color, we screened strains with higher levels of carotenoids productivity in this study. CZ30 showed a stronger red color than $\mathrm{Cz} 5$ and indeed produced more carotenoids than $\mathrm{Cz} 5$. Though the transcriptional levels were higher at $30^{\circ} \mathrm{Cthan}$ at $25^{\circ} \mathrm{C}$, the carotenoid amount was higher at $25{ }^{\circ} \mathrm{C}$. The reason is unclear, but carotenoids productivities may depend on not only the expression levels of carotenoid-biosynthesis genes, but also reaction dynamics between enzymes and substrates, which could be temperature dependent. According to HPLC profile, some new peaks were found and may belong to carotenoids family or carotenoids derivatives for further characterization (Additional file 1: figure S2).

Biorefinery provides a way to efficiently convert renewable biomass to sustainable products, such as biofuels, biochemicals, and biodegradable materials. Plant biomass is considered an abundant resource. However, to facilitate the bioconversion of biomass, pretreatment processes are conducted for lignocellulose destruction, which release various toxic substances and stresses, such as furfurals, which reduce the host performance of bioalcohol fermentation [16]. We showed that an engineered carotenoid-producing $K$. marxianus improved the ability to scavenge free radicals and tolerance to UV damage, furfurals, and ethanol. The enhancement of ethanol tolerance also increased the production of ethanol. In a previous study, K. marxianus showed higher tolerance to harsh environment including furfural stress than $S$. cerevisiae and could be applied to pretreated lignocellulose [9]. Our study supported the potential application of carotenoid producing $K$. marxianus to biofuel production. It also proved the concept of biorefinary to produce ethanol and carotenoid simultaneously. Compared to common industrial yeasts including $S$. cerevisiae and Pichia kluyveri, K. marxianus can produce higher 
branched alcohol, such as isobutanol. The tolerance to isobutanol could make carotenoid producing $K$. marxianus more competitive to produce this next generation biofuel. Furthermore, the tolerance to heavy metal provides an attractive way to utilize phytoremediation biomass from contaminated areas. Improvement of cell tolerance by carotenoids increased the potential of multiple-product conversion and multiple resource utilization.

Carbon flux diversion is an inevitable issue when constructing a host to produce multiple products. In general, minimizing flux diversion of byproducts should be made for maximizing the main production. However, as the tolerance may also enhance the productivity or bio-conversion efficiency, it really depends on what kind of product that a cell factory is intended to produce. As we focused on the protection effect at this stage, we used Cz30 because it yielded $250.5 \mu \mathrm{g} / \mathrm{g}$ of carotenoids whereas Cz5 only yielded $137.2 \mu \mathrm{g} / \mathrm{g}$. In fact, we found that $\mathrm{Cz} 30$ was more ethanol tolerant than Cz5 (Fig. 3d-f).

Natural secondary metabolites have broad pharmaceutical applications, but the yields are usually very low. In order to obtain a sufficient amount of compounds, chemical semi-synthesis provides a way to convert abundant precursors to the valuable products. However, the chemical process often incurs laborious manipulations and organic pollution. Bio-based production through engineered microorganisms provides a sustainable, reliable and efficient way for green production. To convert natural abundant intermediates to functional products could be faster than synthesis from central carbon flux. However, solvent plays a key role to deliver the hydrophobic intermediate to bioprocess, and the cell tolerance to solvent could be a limitation to bioconversion. For instance, paclitaxel has been used for cancer therapy for a long time and bioconversion is thought to be an efficient way for mass production of paclitaxel from abundant 10-deactylbaccatin III. Our carotenoid-producing strain not only improved the tolerance to ethanol but also to the paclitaxel precursor 10-deactylbaccatin III. It could be applied for improving bio-ethanol production and also for paclitaxel bioconversion. This discovery could be potentially applied to paclitaxel biopharmaceuticals industry.

\section{Conclusions}

The engineered carotenoids-producing strain Cz30 showed an enhanced survival rate under the stresses of different toxins such as furfural, arsenate, ethanol, and isobutanol. In addition, the increased tolerance to ethanol allowed the host to deliver more 10-deacetylbaccatin III into the paclitaxel bioconversion process. Thus, our engineered strain $\mathrm{Cz} 30$ has great potential to serve as a chassis cell for bio-refinery.
Table 1 UPL primer sets were used to measure relative quantification of each gene by qRT-PCR

\begin{tabular}{ll}
\hline Primer name & Sequence \\
\hline crtE-UPL\#1-F & CGAGATGCTTTCCCTCCATA \\
crtE-UPL\#1-R & TTCGCTAGGACACGTCAGACT \\
crtl-UPL\#155-F & CCGATCCTTCCTTTTACGTG \\
crtI-UPL\#155-R & CGGCACAAGAATGACGATAG \\
crtYB-UPL\#34-F & CACTGATCTTATCTTTCCCTTATCG \\
crtYB-UPL\#34-R & GTGGTCTCGATAGGCGTCTT \\
tHMG-UPL\#119-F & TTCTGCTATGGCGGGTTC \\
tHMG-UPL\#119-R & GCTGTAACCAAATTCGAAGCA \\
CrBKT-UPL\#159-F & GCTGCTGCAACTGGTTCAC \\
CrBKT-UPL\#159-R & GCACTAGCGGAACTAGCAGAA \\
CZChYb-UPL\#157-F & CGCCCACAAATTACACCATT \\
CZChYb-UPL\#157-R & TCCGAAAAACATACCCCAAG \\
Kan-UPL\#144-F & AGACTAAACTGGCTGACGGAAT \\
Kan-UPL\#144-R & CATCAGGAGTACGGATAAATGC \\
alg9-UPL\#132-F & CAATCAATGGCCGTATCAT \\
alg9-UPL\#132-R & TGTCTCAGAAGCACAGTTTGG \\
\hline
\end{tabular}

\section{Methods}

\section{RT-qPCR quantification}

The RNA was purified by HiQ-Column 12 automated DNA/RNA Purification System (Protech, Taiwan) with an AccuPure Yeast RNA mini kit (AccuBioMed, Taiwan). SuperScript ${ }^{\mathrm{TM}}$ II Reverse Transcriptase (Invitrogen, USA) was used to convert RNA to cDNA. KAPA ${ }^{\mathrm{TM}}$ PROBE FAST qPCR Kit (KAPAbiosystems, USA) and LightCycler 480 (Roche, USA) were conducted for qPCR analysis. The designer UPL (Universal ProbeLibrary, Roche) primer was shown in Table 1 , and $A \lg 9$ was used as a reference gene.

\section{Carotenoid determination}

Yeast was freeze-dried by liquid nitrogen and 2.5 Liter Benchtop Freeze Dry System (FreeZone). Freezedried yeast was homogenized by MagNA Lyser Green Bead (Roche, Basel, Switzerland) and carotenoids were extracted by acetone. Supernatant was than analyzed by reversed phase HPLC (Jasco PU-2089 Quaternary HPLC equipped with Jasco 870-UV intelligent UVVIS). Carotenoid was separated in Nomura Chemical Develosil C30-UG Column, $3 \mu \mathrm{m}$, ID $4.6 \mathrm{~mm} \times \mathrm{L}$ 250 mm - UG17346250 W (Interlink Scientific Services, Sevenoaks, UK) using mobile phase: A buffer, methanol/ MtBE(methyl tert-butyl ether)/Water (81:15:4 vol/vol/ $\mathrm{vol})$, and B buffer, methanol/MtBE/Water (7:90:3 vol/ $\mathrm{vol} / \mathrm{vol}$ ). The elusion gradient was set as $100 \% \mathrm{~A}$ at $0 \mathrm{~min}$, followed by linear gradient to $100 \% \mathrm{~B}$ at $50 \mathrm{~min}$, and returned to $100 \% \mathrm{~A}$ at $60 \mathrm{~min}$. Carotenoids were identified and quantified by $460 \mathrm{~nm}$ absorbance. The accumulation 
of carotenoids in an engineered strain was estimated as the total amount of carotenoids: Car $(\mathrm{mg} / \mathrm{g})=4.69 \mathrm{~A}_{440} \mathrm{x}$ acetone $\mathrm{ml} /$ cell weight $\mathrm{g}$. [19].

\section{Antioxidant capacity assay}

After $72 \mathrm{~h}$ of culturing in YPG medium (containing 1\% yeast extract, $2 \%$ peptone, and $2 \%$ galactose) at $25{ }^{\circ} \mathrm{C}$, the cells were lyophilized for extraction and analysis. Antioxidant capacity assays of $K$. marxianus wild type (WT) and engineered strains $\mathrm{Cz} 30$ were conducted with the ABTS substrate reaction and Trolox Equivalent Antioxidant Capacity (TEAC) assay. ABTS solution was prepared by $4.67 \mathrm{mM}$ ABTS radical cation (Sigma-Aldrich) and $2.45 \mathrm{mM}$ potassiumpersulfate and keep in dark. The assay was performed by mixing ABTS $(990 \mu \mathrm{l})$ with analytes $(10 \mu \mathrm{l})$, and the decline of $734 \mathrm{~nm}$ absorbance was represented antioxidant ability. Trolox is an analog of vitamin $\mathrm{E}$ and TEAC is use trolox equivalent as a benchmark for different antioxidants.

\section{UV exposure assay}

Kluyveromyces marxianus WT and Cz30 were tested for survival under UV light exposure. The cell pellets were harvested and exposed to UV light (Viber Lourmat, TFX$20 \mathrm{M}, 6 \times 15 \mathrm{~W}$ ) for 5,10 , or $20 \mathrm{~min}$, and then dropped into the YPG plate (containing $1 \%$ yeast extract, $2 \%$ peptone, $2 \%$ galactose, and $2 \%$ agar) with a series dilution culturing for $72 \mathrm{~h}$ at $25^{\circ} \mathrm{C}$.

\section{Stress tolerance assays}

The engineered yeasts were subjected to tolerance tests in YPG medium (containing 1\% yeast extract, 2\% peptone, and $2 \%$ galactose) with initial OD of 1.0 and different initial concentrations of chemicals, including furfural, ethanol, isobutanol, and 10 deacetylbaccatin III. After $24 \mathrm{~h}$, survival tests were conducted in YPG plates with a series dilution culturing at $25{ }^{\circ} \mathrm{C}$ for $72 \mathrm{~h}$. The growth rate was measured by $600 \mathrm{~nm}$ absorbance at different time points. The growth density experiment was repeated three times and plate assay was performed one time.

\section{Ethanol production}

Cells were grown on YPG medium (containing 1\% yeast extract, $2 \%$ peptone, and $20 \%$ galactose) and the total production of ethanol was analyzed by HPLC (Jasco PU-2089 Quaternary HPLC pump, JASCO International Co., Tokyo, Japan) with thICSep ICE-COREGEL 87H3 Column (Transgenomic, Nebraska, USA) and Shodex RI-101 Refractive Index Detector (ECOM, Praha, Czech Republic). Each experiment was repeated three times.

\section{Supplementary information}

Supplementary information accompanies this paper at https://doi. org/10.1186/s12934-019-1205-y.

Additional file 1. Additional figures.

\section{Abbreviations}

ROS: reactive oxygen species; UV: ultraviolet; PGASO: Promoter-based Gene Assembly and Simultaneous Overexpression; RT-qPCR: quantitative reverse transcription PCR; ABTS: 2,2'-azino-bis (3-ethylbenzothiazoline-6-sulphonic acid); TEAC: Trolox Equivalent Antioxidant Capacity; As[V]: arsenate; 10-DB III: 10-deacetyl baccatin III; YPG: medium containing peptone, yeast extract and galactose.

\section{Acknowledgements}

This work was supported by the Ministry of Science and Technology, Taiwan [MOST 107-2311-B-039-002-, MOST 107-2621-M-039-001-, and MOST 1062622-8-005-003-TB1], Academia Sinica, China Medical University Hospital [DMR-106-120 and DMR-107-112], and National Chung Hsing University [The ENABLE Center]. This work was also supported by the "Innovation and Development Center of Sustainable Agriculture" from The Featured Areas Research Center Program within the framework of the Higher Education Sprout Project by the Ministry of Education (MOE) in Taiwan.

\section{Authors' contributions}

$\mathrm{H}-\mathrm{LL}$ and CT performed the experiments, analyzed the data; J-JC and H-LL designed the study and analyzed the data. Y-JL provided technical advice and participated in data interpretation. H-LL drafted the manuscript. W-HL and $\mathrm{C}-\mathrm{CH}$ coordinated and advised the study and revised the manuscript. All authors read and approved the final manuscript.

\section{Availability of data and materials}

The datasets used in this study are available from the corresponding author on request.

All data generated or analyzed during this study are included in this published article. There is no additional material.

Ethics approval and consent to participate Not applicable.

\section{Consent for publication}

Not applicable.

\section{Competing interests}

The authors declare that they have no competing interests.

\section{Author details}

${ }^{1}$ Ph.D. Program in Microbial Genomics, National Chung Hsing University and Academia Sinica, Taipei, Taiwan. ${ }^{2}$ Department of Medical Research, China Medical University Hospital, No. 91 Hsueh-Shih Road, Taichung 402, Taiwan.

${ }^{3}$ Biodiversity Research Center, Academia Sinica, 128 Academia Road, Sec. 2, Nankang, Taipei 115, Taiwan. ${ }^{4}$ Department of Life Sciences, National Chung Hsing University, No. 250, Kuo Kuang Rd, Taichung 402, Taiwan. ${ }^{5}$ Innovation and Development Center of Sustainable Agriculture, National Chung Hsing University, No. 145, Xingda Rd, South Dist, Taichung 402, Taiwan. ${ }^{6}$ Department of Ecology and Evolution, University of Chicago, Chicago, IL 60637, USA.

Received: 6 May 2019 Accepted: 3 September 2019

Published online: 10 September 2019

\section{References}

1. Lynd LR, van Zyl WH, McBride JE, Laser M. Consolidated bioprocessing of cellulosic biomass: an update. Curr Opin Biotechnol. 2005;16:577-83.

2. Fatih Demirbas M. Biorefineries for biofuel upgrading: a critical review. Appl Energy. 2009;86:S151-61. 
3. Martinez-Hernandez E, Sadhukhan J, Campbell GM. Integration of bioethanol as an in-process material in biorefineries using mass pinch analysis. Appl Energy. 2013;104:517-26.

4. Kitagaki H, Araki Y, Funato K, Shimoi H. Ethanol-induced death in yeast exhibits features of apoptosis mediated by mitochondrial fission pathway. FEBS Lett. 2007;581:2935-42.

5. Kim D, Hahn JS. Roles of the Yap1 transcription factor and antioxidants in Saccharomyces cerevisiae's tolerance to furfural and 5-hydroxymethylfurfural, which function as thiol-reactive electrophiles generating oxidative stress. Appl Environ Microbiol. 2013;79:5069-77.

6. Lin KH, Chien MF, Hsieh JL, Huang CC. Mercury resistance and accumulation in Escherichia coli with cell surface expression of fish metallothionein. Appl Microbiol Biotechnol. 2010;87:561-9.

7. Chin WC, Lin KH, Chang JJ, Huang CC. Improvement of n-butanol tolerance in Escherichia coli by membrane-targeted tilapia metallothionein. Biotechnol Biofuels. 2013;6:130.

8. Chin WC, Lin KH, Liu CC, Tsuge K, Huang CC. Improved n-butanol production via co-expression of membrane-targeted tilapia metallothionein and the clostridial metabolic pathway in Escherichia coli. Biotechnol Biofuels. 2017;17:36.

9. Chang JJ, Ho CY, Mao CT, Barham N, Huang YR, Ho FJ, Wu YC. A thermoand toxin-tolerant kefir yeast for biorefinery and biofuel production. Appl Energy. 2014;132:465-74.

10. Chang JJ, Thia C, Lin HY, Liu HL, Ho FJ, Wu JT, Shih MC, et al. Integrating an algal $\beta$-carotene hydroxylase gene into a designed carotenoid-biosynthesis pathway increases carotenoid production in yeast. Bioresour Technol. 2015;184:2-8

11. Stahl W, Sies H. $\beta$-Carotene and other carotenoids in protection from sunlight. Am J Clin Nutr. 2012;96:1179S-84S.

12. Kidd P. Astaxanthin, cell membrane nutrient with diverse clinical benefits and anti-aging potential. Altern Med Rev. 2011;16:355-64.
13. Hussein G, Sankawa U, Goto H, Matsumoto K, Watanabe H. Astaxanthin, a carotenoid with potential in human health and nutrition. J Nat Prod. 2006:69:443-9.

14. Prasad MNV. Phytoremediation crops and biofuels. In: Lichtfouse E, editor. Sustainable agriculture reviews. Springer: Cham; 2015. p. 159-261.

15. Chang JJ, Ho CY, Ho FJ, Tsai TY, Ke HM, Wang CH, Chen HL, et al. PGASO: a synthetic biology tool for engineering a cellulolytic yeast. Biotechnol Biofuels. 2012;5:53.

16. Iwaki A, Kawai T, Yamamoto Y, Izawa S. Biomass conversion inhibitors furfural and 5-hydroxymethylfurfural induce formation of messenger RNP granules and attenuate translation activity in Saccharomyces cerevisiae. Appl Environ Microbiol. 2013;79:1661-7.

17. Wysocki R, Tamás MJ. Saccharomyces cerevisiae as a model organism for elucidating arsenic tolerance mechanisms. In: Banfalvi G, editor. Cellular effects of heavy metals. Dordrecht: Springer; 2011. p. 87-112.

18. Malik S, Cusidó RM, Mirjalili MH, Moyano E, Palazón J, Bonfill M. Production of the anticancer drug taxol in Taxus baccata suspension cultures: a review. Process Biochem. 2011;46:23-34.

19. Porra RJ, Thompson WA, Kriedemann PE. Determination of accurate extinction coefficients and simultaneous equations for assaying chlorophylls a and b extracted with four different solvents: verification of the concentration of chlorophyll standards by atomic absorption spectroscopy. Biochimica et Biophysica Acta (BBA)-Bioenergetics. 1989:975:384-94

\section{Publisher's Note}

Springer Nature remains neutral with regard to jurisdictional claims in published maps and institutional affiliations.
Ready to submit your research? Choose BMC and benefit from:

- fast, convenient online submission

- thorough peer review by experienced researchers in your field

- rapid publication on acceptance

- support for research data, including large and complex data types

- gold Open Access which fosters wider collaboration and increased citations

- maximum visibility for your research: over $100 \mathrm{M}$ website views per year

At BMC, research is always in progress.

Learn more biomedcentral.com/submissions 